\title{
Development of Orthopaedics in Kenya
}

\author{
J.A.O. Mulimba, MBChB, MMed, FRCS, Associate Professor, Consultant Orthopaedic and Trauma Surgeon, \\ Department of Orthopaedic Surgery, College of Health Sciences, University of Nairobi, \\ P.O.Box 52507-00200, Nairobi, Kenya,Email:prof-jao@uhmc.co.ke
}

\section{INTRODUCTION}

It is difficult to say at what point Orthopaedics in Kenya broke away from general surgery. All over the world, orthopaedics has developed as an off shoot of general surgery. There are various forms of practice of orthopaedics i.e. as part of trauma or totally divorced from trauma. In Kenya, orthopaedics is practiced together with orthopaedic trauma. This has really developed on the British model. Here an attempt is made to put on record what is known about orthopaedics in Kenya to pave the way for further research into the history of orthopaedics in this country. The development of orthopaedics will be traced along three pathways i.e. human resource, orthopaedic education and orthopaedic practice.

\section{HUMAN RESOURCE}

Orthopaedic and trauma surgery was first carried out by general surgeons. The first recorded orthopaedic surgeon at then King George Hospital was Bill Kirkaldy Willis, who was a Canadian in origin. There is no evidence that he made any effort to increase the number of orthopaedic surgeons in the country at the time, perhaps there was no need then. The first recorded indigenous african orthopaedic surgeon is Dr. Micah Majale who was trained in France. He reported on duty at the KNH hospital in 1965 . He was then joined by Mr. S. K. Suleiman and later M. A. Sheikh and J. Bodo as the 2nd African orthopaedic surgeon. There were other surgeons who joined in but left early to go into private practice and these included J. Beecher, D. Stuart and G. Sikalieh. Majale passed away in 1976, Suleiman left for Australia, Canada, Pakistan then Aga Khan University Hospital, Nairobi and now gone again. Sheikh left for private practice in Nairobi Hospital and is now retired in USA.

Beecher after private practice in Nairobi hospital left for Australia and is now deceased.

Stuart, after private practice in Nairobi hospital has now retired to the coast resort town of Mombasa. Mr. Bodo went into private practice at the Aga Khan
University Hospital. Sikalieh after going into private practice passed away in 2006. The current crop of orthopaedic surgeons at $\mathrm{KNH}$ are many but suffice it to mention some of the older ones who include F. Hagembe, S. Kuria, C. Nyakinda, C. Odongo, J. Mogire, F. Otsyeno, G. Kibuga, O. Afulo, R. Ombachi, K. Gichuhi, J. Kirugo, G. Gikenye, J. Ochieng and many others.

While the government was developing orthopaedic personnel at $\mathrm{KNH}$ and elsewhere, the University of Nairobi came into being with the school of medicine established in 1967. The first orthopaedic lecturer was Mr.J.C.T.Church.The department of orthopaedic surgery came into being in 1972 with its only lecturer at the time being Dr.M.K. Jeshrani. The first orthopaedic professor was A. Bencivenga appointed in 1973 from Somalia. He was a urological surgeon turned orthopaedic trauma surgeon. It is true that he introduced AO surgery in Kenya. One may disagree with some of his doctrines but he was a stickler to perfection and good at what he did. Prof. Bencivenca took over from Mr. Dennis Wainwright who run the department for sometime and was responsible for establishment of a link between the department of orthopaedic surgery of University of Nairobi and the Robert Jones and Agnes Hunt Orthopaedic Hospital at Oswestry, England which trained a number of surgeons including Bodo, Sheikh, Mulimba, Gakuu, Atinga, Mwangi, Museve etc. The first african indigenous head of the department was Prof. B.S. Mbinyo who took over in 1983. Other heads have included Prof. J.A.O. Mulimba (1989-1995), Prof. J.E.O. Atinga (1995-2001) and currently Prof. L.N. Gakuu (2001-2007). Others who have passed through the department include Mr. Brambatt, K.P. Mishra, Prof. Bovile, P.M. Heda, A. Ndumbu, P. Oroko, V. Kireti, G.G. Kamau. Those still in the department include Drs. J.C. Mwangi, G.K. Museve, V. Mutiso, C. Oburu, T.S. Mogire and E.M. Gakuya.

There is a small number of surgeons engaged only in private practice and include S. Owinga,T.Byakika and K.J. Shah. Whereas most of the orthopaedic surgeons have been and are in Nairobi a few have been outside Nairobi. Mombasa had the late MacVicker, H. Patel, E. Ambeva and now Sheth. There is a single Egyptian 
orthopaedic surgeon in Nakuru, Salah Amin and Dr. L. Lelei in Eldoret at the Moi University medical school. There are a number of orthopaedic surgeons in some mission hospitals including Tenwek, Kikuyu and Kijabe. Most have been visiting Americans on short stay but there are some indigenous orthopaedic surgeons such as J. Murila, King'ori, Macharia, Muteti and others.

It should be noted that inspite of that large number of orthopaedic surgeons, most of the orthopaedic trauma cases in the country are still managed by medical officers and general surgeons. The quality of work so produced is a different matter.

\section{ORTHOPAEDIC EDUCATION}

By World Health Organisation (WHO) recommendations, there should be one orthopaedic surgeon per 100,000 population. The number of orthopaedic surgeons in Kenya is estimated at sixty. Those registered as such in the current Medical Practitioners and Dentist Board (MP\&DB) register are 31. This gives a ratio of 1: 550,000. Only two provincial hospitals, two district and four mission hospitals have an orthopaedic surgeon. Since its breakway from general surgery in 1972, the department of orthopaedic surgery has been responsible for the training of orthopaedic and orthopaedic trauma surgeons.

Currently medical students learn orthopaedics in their $3^{\text {rd }}$ and $5^{\text {th }}$ years. They do 12 weeks in third year and 6 weeks in $5^{\text {th }}$ year. Weekly lectures are given to fifth year students throughout the academic year. Postgraduate students in surgery do three months in casualty, and two unit rotations of three months each. Those who have finished their general surgical training and want to become orthopaedic surgeons then get attached to the orthopaedic service at $\mathrm{KNH}$ for one to two years then go out of the country to United Kingdom or South Africa for further training. A number do not come back. A curriculum has been worked out and is going through the works for a masters in orthopaedic surgery of the University of Nairobi similar to the courses run in Uganda and Tanzania. Currently the younger crop of orthopaedic surgeons have been trained in Uganda. The paramedicals in the orthopaedic field are all trained at the Kenya Medical Training Centre.

The other training avenue of late has been through the college system of College of Surgeons of East,Central and Southern Africa (COSECSA). This looks like it may be the best alternative, but unfortunately the college is going through a lean period financially at the moment. Another boost is the creation of the Kenya Orthopaedic Association (KOA). The KOA which started off as The Nairobi Orthopaedic club, is going to play a major roll in continuing professional development, planning of orthopaedic training together with various training institutions, deciding through the MP\&DB who should be registered as an orthopaedic surgeon (Criteria have already been drawn) and laise with external orthopaedic bodies. KOA has also launched a journal which it is hoped will be a major contributor to dissemination of knowledge in Kenya and beyond in the region. KOA is also working out mechanism of working with industry to promote Biomedical Engineering, instrument and implant manufacture.

\section{ORTHOPAEDIC PRACTICE}

Orthopaedic surgery had humble beginnings as a small section of general surgery. It had a few beds assigned to orthopaedic trauma and minor orthopaedics including minor operations for club foot, acute and chronic osteomyelitis, excision of ganglions and exostoses, amputations and others. The operations grew in complexity as orthopaedic surgeons started to appear on the scene with osteotomies, laminectomies and hemiarthroplasties being done. Later more complex surgery came into being including total arthroplasties of hips and knees, complex spinal surgery, arthroscopies and arthroscopic reconstructions, fixation of complex fractures among many surgeries.

\section{CURRENT SITUATION}

The current situation is that trauma still takes most of the ward space and operating time in orthopaedic service. The tragedy is that no wards have as yet been allocated to cold orthopaedics. The wards are very crowded, for compound fractures surgical toilets are still done late with consequent high infection rate as reported by Gakuu. The teaching institutions are still poorly staffed and mostly by juniors. It is noteworth that the largest teaching hospital in the country the orthopaedic department has no offices, laboratories and other related facilities. Lack of specialisation in other related specialities also affects quality of work namely orthopaedic radiology and orthopaedic pathology. Consequently, clinicopathological conferences are few and far between. It is good to note that with the advent of KOA more specialised workshops are now 
being held. Lone ranger management methods are still the norm. This affects orthopaedic practice in two ways, there are individual management methods for common problems instead of protocols. Taking holidays is difficult as one usually has to close office. Whereas the number of orthopaedic surgeons is increasing, there is still slow pace in specialisation, and even those who have specialised, for economic reasons, hardly stick to their specialities. The distribution of orthopaedic service is still quite uneven leaving poor service to be given to those in far off areas. There has been reluctance in some surgeons to go out for seminars, conferences and workshops, the MP\&DB is certainly seeing to it that this improves. As a result of lack of funds research and publications have been lacking. The problem of litigation has caught up with medical practice in Kenya, and with orthopaedics in particular. "Doctor finish doctor" mentality has contributed a lot to this problem.

\section{Way forward}

I see a great future for orthopaedics in Kenya. The many things that will need to be done include encourage specialization; establish separate orthopaedic and orthopaedic trauma centres. Other orthopaedic centres will have to be established out of the traditional orthopaedic centres. There is need to encourage cooperation between other centres and centres in developed countries such as occurs between $\mathrm{KNH}$ and Fort Wayne; Eldoret's Moi University and Indianapolis both in USA. The population will need to be educated on available orthopaedic services and be encouraged to use them. Exchange of ideas will have to be encouraged through Continuing Professional Development (CPD) and publication in the KOA launched journal and other journals. Petty jealousies leading to increased litigation will need to be discouraged.

Training institutions will have to start aggressive orthopaedic training programmes. Together with this, relevant research will have to be carried out. Money will only become available if evidence is shown of willingness to do research as evidenced by research protocols. Orthopaedic service will have to be involved in national programmes by providing leadership in relevant fields such as road traffic accidents and disaster management. There is need for development of orthopaedic surgical practice by collaboration of the Ministry of Health, training institutions, KOA and MP\&DB. There is along way yet to go, but a start has been made.

\section{REFERENCES}

1. Loefler I.P.J. The history of surgery in Eastern Africa. Pro. Assoc. Surg. East Afr. 1988; 11: 55-59.

2. Surgery in East Africa:Technology and Training. Pro. Assoc. Surg. East Afr. 1981; 4: 58-156.

3. Kenyatta National Hospital: A hundred years of quality health care,1901-2001.

4. Kenyatta National Hospital: Strategic Plan, 2005-2010.

5. Gakuu L.N. Surgical wound infections: Their causes and management. East Afr. Med. J. 2005; 82: 329-330.

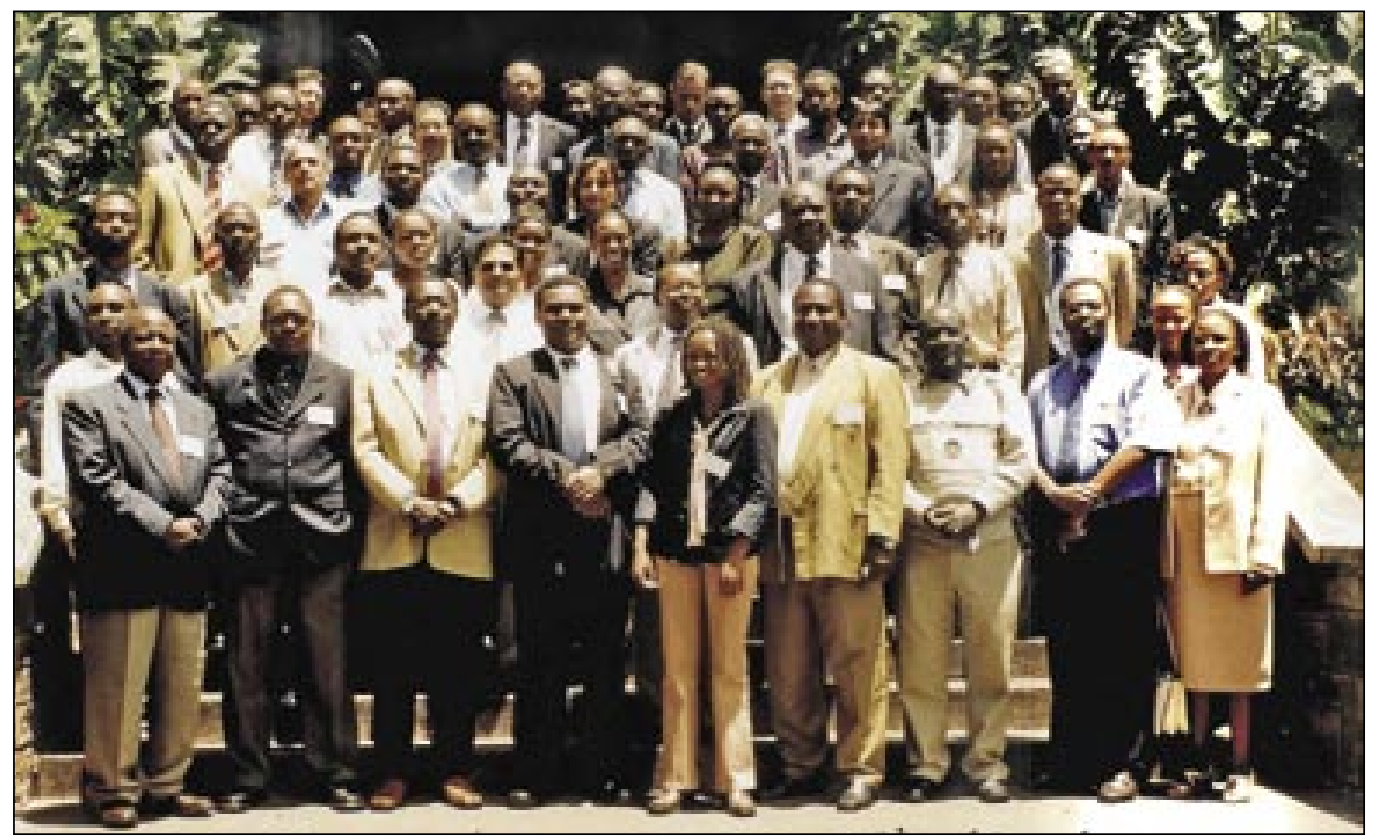

Figure 1: The first KOA annual general meeting at the Panafric Hotel on 23 $3^{\text {rd }}$ March 2006. 